\title{
Demokratie und Bildung
}

\section{Politische Erwachsenenbildung unter Pädagogikstudierenden in Polen}

\author{
Błażej Przybylski
}

Eingegangen: 27. März 2017 / Angenommen: 25. Mai 2017 / Online publiziert: 21. Juni 2017

(C) Der/die Autor(en) 2017. Dieser Artikel ist eine Open-Access-Publikation.

Zusammenfassung Der vorliegende Artikel stellt die Ergebnisse einer Untersuchung des Autors zu (bildungs-)politischen Ansichten von polnischen PädagogikStudierenden vor. Die Untersuchung erfolgte im Anschluss an die jüngsten Parlamentswahlen in Polen (2015), bei denen die Partei „Prawo i Sprawiedliwośćc (,Recht und Gerechtigkeit“, PiS) die absolute Mehrheit gewonnen hatte. Das Wahlprogramm dieser Partei kündigte radikale Änderungen an, ebenfalls im Bildungswesen. Das Ziel der Studie war die Erkundung des Interesses an Politik, des Verhältnisses zur Demokratie, der allgemeinen Bildungsorientierungen und der Ansichten der Studierenden zu den Plänen der neuen Regierungspartei im Bildungssystem. Im Ergebnis zeigten sich Diskrepanzen zwischen den allgemeinen Bildungsvorstellungen (eher links und liberal) zu den konkreten Absichten und ersten Veränderungen der Partei (eher rechts und konservativ). Dieser Befund gibt Grund, die Ziele und Methoden politischer Erwachsenenbildung in Polen, aber auch in anderen europäischen Ländern zu überprüfen.

Schlüsselwörter Bildung $\cdot$ Demokratie $\cdot$ Ideologie $\cdot$ Pädagogik $\cdot$ Bildungsreformen

\section{Democracy and education}

Civic education among polish pedagogy students

\begin{abstract}
The article presents the results of the author's study on the views of pedagogy students on politics and education. The research has been undertaken subsequently to the 2015 parliamentary elections won by the party "Prawo i Sprawiedliwość" ("Law and Justice", PiS). During the election campaign, this party's program offered radical changes, also in the Polish educational system. The aim of
\end{abstract}

B. Przybylski, PhD (ه)

The Maria Grzegorzewska University, Szczęśliwicka 40, 02-353 Warsaw, Polen

E-Mail: blazej.przybylski@wp.pl 
the study was the recognition of the level of interest in politics, the approach to democracy and education as well as the students' relation to the solutions in the educational system proposed by the winning party. The result shows discrepancies between general views on education (rather lefty and liberal) and the first modifications of the party (rather right-wing and conservative). This finding gives reason to verify the aims and methods of political education of adults in Poland, as well as in other European countries.

Keywords Education - Democracy · Ideologies · Pedagogy students · Educational reforms

\section{Demokratie und politische Bildung}

Demokratie kann nur gelingen, wenn die Mitglieder einer demokratischen Gemeinschaft ihre Werte mittragen und in der Lage und willens sind, am demokratischen Geschehen teilzuhaben. Entsprechend wird politische Bildung definiert - nicht nur in Deutschland: Sie soll das Verständnis für politische Sachverhalte stärken, das demokratische Bewusstsein festigen und zur politischen Mitarbeit befähigen (BMI 2001). Eine zentrale Aufgabe für das konstruktive Fortbestehen der Gesellschaftsform „Demokratie“, denn, in den Worten von Oskar Negt: „Demokratie ist die einzige politisch verfasste Gesellschaftsordnung, die gelernt werden muss - immer wieder, täglich und bis ins hohe Alter hinein“ (Negt 2010, S. 174).

Natürlich gibt es unterschiedliche Auffassungen darüber, was und wie es gelernt werden muss. So gibt es Vorschläge, die politische Bildung von den Institutionen und der konkreten Politik zu entkoppeln, solche, die Prinzipien der freiheitlich demokratischen Ordnung zu vermitteln oder aber solche, ,die Kritikfähigkeit wieder als zentrale Kategorie politischer Bildung“" zu begreifen (Ahlheim und Mathes 2005). In der diskursiven Auseinandersetzung um den richtigen Weg der politischen Bildung zeigen sich Kontroversen auch zwischen den Generationen (Brandt et al. 2003).

Gerade heute stellt sich die drängende Frage, wie es mit der politischen Bildung bestellt ist, wenn man die Entwicklungen des Populismus, des Nationalismus und der Autokratie in Ländern wie Russland, USA, Frankreich und Türkei, aber auch Deutschland (AfD) bedenkt. Auf welchen gesellschaftlichen Faktoren beruhen solche Entwicklungen, welche Rolle spielen dabei die Einstellungen der Bevölkerung zur Demokratie und wie kann politische Erwachsenenbildung damit umgehen?

Von besonderer Bedeutung ist diese Frage in starken Umbruch- und Veränderungssituationen, da dann immer weitreichende Konsequenzen damit verbunden sein können. Wir beschäftigen uns hier mit politischen Einstellungen in einer solchen Situation, den tiefgreifenden Veränderungen in Polen seit der letzten Wahl vor zwei Jahren. Wir beschränken uns auf Einstellungen zur Demokratie im Allgemeinen sowie zur Bildung im Grundsatz und in konkreten Einzelfragen.

Ziel des vorliegenden Artikels ist es, die Ansichten von Pädagogik-Studierenden zur Bedeutung von Demokratie, ihr Interesse an Politik sowie ihre Auffassung über Bildung zu erkunden. Wir fragen auch nach den Ansichten über konkrete Neuregelungen im Bildungsbereich seit den letzten Wahlen. Das Wissen um die Ansichten 
und Einstellungen gerade dieser Studierenden hat eine erhebliche Relevanz im Bildungsbereich, weil diese höchstwahrscheinlich in der Zukunft lehrend tätig sein oder andere Positionen bekleiden werden, die mehr oder weniger stark mit dem Bildungssystem verbunden sind. Sie sind, wenn man so will, als Verantwortliche für die Vermittlung von Wissen und Bewusstsein an künftige Generationen Schlüsselfiguren in der Entwicklung einer demokratischen Gesellschaft. Es ist eine wichtige Aufgabe, diese Personengruppe politisch zu bilden. Unsere Untersuchungsfrage lautete daher: ,Wo liegen Ansatzpunkte politischer (Erwachsenen-)Bildung in den Ansichten der Pädagogik-Studierenden zu Demokratie und Bildung?" In diesem Aufsatz präsentieren wir einige wichtige Ergebnisse unserer Forschungsarbeit.

\section{Der politische Kontext in Polen}

Im Jahr 2015 hat nach zwei Amtszeiten einer Mitte-Rechts-Regierung aus Bürgerplattform und der Polnischen Bauernpartei die nationalkonservative Partei „Recht und Gerechtigkeit" (PiS) bei der Wahl die absolute Mehrheit im polnischen Parlament (Sejm) gewonnen. Im selben Jahr gewann auch der von derselben Partei aufgestellte Kandidat Andrzej Duda die Präsidentschaftswahl. Seit dem Sturz der kommunistischen Volksrepublik Polen im Jahr 1989 sind die Nationalkonservativen die einzige politische Kraft, die eine Alleinregierung zustande gebracht hat. Ihr Wahlkampf stand unter dem Motto „Guter Wandel“. Zum ersten Mal zogen keinerlei linke Gruppierungen in das Parlament Polens ein.

Die PiS hat bereits viele radikale Veränderungen in weiten Bereichen des öffentlichen Lebens in Polen angekündigt - und teilweise bereits umgesetzt. Die Tatsache, dass alle Macht in den Händen einer Partei konzentriert ist, die ein eindeutiges ideologisches Profil aufweist und zudem die Entwicklung der vergangenen Jahre in Polen sehr kritisch sieht (der aktuelle Zustand des Landes galt ihr als katastrophal, was in einer der wichtigsten Wahlkampfparolen, „Polen in Trümmern“, zum Ausdruck kam), hat zu Sorgen hinsichtlich Demokratie und Rechtstaatlichkeit geführt. Die bereits umgesetzten Reformen, von den politischen Gegnern der PiS „Deformen“ genannt, haben zu heftigen Auseinandersetzungen geführt - etwa die Maßnahmen gegenüber den öffentlichen Medien und dem Verfassungsgericht. Zu den Bereichen, die grundsätzlich verändert werden sollen, zählt auch die Bildung. Viele Wahlversprechen von PiS betrafen direkt das Bildungssystem.

Nach dem Machtantritt der PiS-Regierung ist die Gesellschaft zunehmend polarisiert. Ideologische Konflikte vermehren und verstärken sich, zudem werden sie von immer heftigeren Emotionen begleitet. Dem Sozialpsychologen Janusz Reykowski (2013, S. 19) zufolge ,haben sich in Polen zwei Lager mit gegensätzlichen ideologischen Überzeugungen herausgebildet“. Der ehemalige Dissident Adam Michnik (2016, S. 10) spricht davon, dass ,die wirkliche Kluft heute zwischen der Anhängerschaft einer offenen und einer geschlossenen Gesellschaft verläuft“. Diese Teilung betrifft Schlüsselthemen wie die Beziehung zum Anderssein (andere Nationen, Kulturen, sexuelle Orientierung, Glaubensbekenntnisse), das Verhältnis von Staat und Kirche, das Wesen der Demokratie, die Achtung von Minderheitsrechten und die Position Polens in Europa. 
Die PiS-Regierung vollzieht nach Meinung eines bedeutenden Teils der polnischen Gesellschaft eine Abkehr von demokratischen Werten. Unter Berufung auf den Willen des Souveräns und den Gewinn demokratischer Wahlen implementiert die Regierungspartei zusammen mit ihrem Präsidenten radikal ihr eigenes Programm und zerstört dabei eine demokratische Institution nach der anderen. Was die Rechtsstaatlichkeit der Regierungsmaßnahmen angeht, so haben nicht nur Juristen und Juristinnen, sondern auch die internationale Meinung hierbei ihre Zweifel. Prominente polnische Oppositionelle der früheren Solidarność-Bewegung befürchten, dass die Demokratie in Polen bedroht ist, manche sprechen gar von ihrem Ende. Der (bereits ehemalige) Präsident des Verfassungsgerichtshofes Andrzej Rzepliński - der vielen als ein Symbol des unnachgiebigen Kampfes für die Achtung des Rechts und der Unabhängigkeit der Gerichte gilt, von der PiS hingegen als politischer Gegner betrachtet wird, der den „Guten Wandel“ behindern will - sagte in einem Kommentar zur amtierenden Regierung, dass ,,individuelle Herrschaft ohne jede Kontrolle und bei vollständiger Ignoranz der Verfassungsorgane eine Form der Alleinherrschaft darstellt“" (Rzepliński 2017).

In den ersten Wochen des Jahres 2017 erschienen mindestens zwei internationale Berichte, die die zunehmend mangelhafte Gestalt der polnischen Demokratie anzeigen. Laut dem Bericht „Demokratie-Index“ des Wochenblatts „The Economist“ hat Polen 2016 im Vergleich zum Vorjahr vier Plätze verloren; dem Land wurde der 52. Platz zuerkannt (Intelligence Unit 2016). Dies ist das schlechteste Ergebnis seit 2006, als „The Economist“" mit der Veröffentlichung seiner jährlichen Berichte über den Zustand der Demokratie begann. Bereits im Jahre 2015, nach dem Doppelsieg der PiS in den Präsidentschafts- und Parlamentswahlen, war Polen in dieser Rangliste um acht Positionen auf die 48. Stelle abgerutscht. Das Expertenteam, das die Studie erstellt hat, verwies darauf, in welchem Maße die Beobachtenden aus der Europäischen Union über das Tempo erschüttert sind, mit dem die PiS im Namen der moralischen Erneuerung und einer Reform des Staates Veränderungen in den öffentlichen Medien und beim Verfassungsgericht durchführt.

Wie polnische Medien unter Verweis auf die „Deutsche Welle“ berichteten, prangerte auch die Organisation „Reporter ohne Grenzen“ die ,eklatante Verletzung der Pressefreiheit durch die polnische Regierung und antideutsche Tendenzen“ an. In der jährlichen Rangliste zur Pressefreiheit fiel Polen im Jahr 2016 um 29 Plätze auf Rang 47 (Online report 2017). Nicht nur ein Teil der polnischen Gesellschaft ist besorgt über den Zustand der polnischen Demokratie unter der Regierung von PiS, sondern auch die internationale Meinung und transnationale Organisationen erkennen hier reale Gefahren. Bisher war angenommen worden, dass Polen zwar eine schwache Zivilgesellschaft, dafür aber starke Institutionen besitzt, die ein Garant für die Aufrechterhaltung eines vollständig demokratischen Systems seien. Die jüngsten Veränderungen in den öffentlich-rechtlichen Medien und beim Verfassungsgericht sowie die Versuche zur Einschränkung der Versammlungsfreiheit und die Kontrolle von Nichtregierungsorganisationen zeigen jedoch, dass das demokratische System ohne eine starke Zivilgesellschaft nicht effizient funktionieren kann.

Dem Komplex „Bildung“ widmete die PiS in ihrem Wahlprogramm viel Platz der Schule etwa wurde vorgeworfen, die Wachstumspotenziale der polnischen Gesellschaft nicht entsprechend zu nutzen. Die Partei versprach spezifische Lösungen, 
welche die Situation der Schulbildung verbessern sollen. Bereits im ersten Absatz des Programms von PiS fand sich die Definition einer effektiven Schule und ihrer Aufgaben: „Eine gute Schule - in einem grundsätzlichen Verständnis - dient der Vermittlung von Kenntnissen und Fähigkeiten, gestaltet das Denken, führt junge Menschen in den kulturellen Code ein, den die polnische Nation in seiner Geschichte hervorgebracht hat, erzieht zum Leben in der Gemeinschaft und fördert angemessenes moralisches und bürgerschaftliches Verhalten“ (Prawo i Sprawiedliwość 2014, S. 129). Die vorrangige Funktion der Erziehung solle in der Vermittlung traditioneller Werte liegen: „Die Absolventen einer polnischen Schule sollten über einen gemeinsamen Wissensschatz verfügen sowie die gemeinsamen Symbole, Referenzen und Vorstellungen kennen, die der polnischen Identität zugrunde liegen und die Erfahrung unserer Nation bilden“ (ebd., S. 130). Die Herausbildung entsprechender Denkformationen soll hierbei der Garant für die Entwicklung des Landes sein. Die Schule solle nur die eine, richtige Wahrheit überliefern und entsprechende Verhaltensweisen im Bereich von Sitten und Moral lehren.

\section{Die methodische Anlage der Untersuchung}

Das Ziel der Studie war es erstens, Ansichten zu den Fragen von Demokratie in der aktuellen politischen Debatte in Polen zu ermitteln. Aus verschiedenen, vor allem auch aus pragmatischen Gründen haben wir diese Ansichten bei Studierenden der Maria-Grzegorzewska-Universität in Warschau (Akademia Pedagogiki Specjalnej, im Folgenden APS genannt) ermittelt. Die Forschungsfrage lautete: Wo liegen Ansatzpunkte politischer (Erwachsenen-)Bildung in den Ansichten der Pädagogikstudierenden zu Demokratie und Bildung? Als die wichtigsten Aspekte der Studie galten:

1. Wie groß ist das Interesse der Studierenden an Politik?

2. Wie ist das Verhältnis der Studierenden zur Demokratie?

3. Was ist die Ansicht der Studierenden über Bildung?

4. Wie stehen die Studierenden zu konkreten Entscheidungen zum Bildungswesen?

Zur Beantwortung der Fragenkomplexe 1, 2 und 4 diente ein von mir speziell für diese Studie erarbeiteter Fragebogen, der aus 16 geschlossenen Fragen bestand. Zur Ermittlung der Bildungsorientierungen der Studierenden (insbesondere Fragenkomplex 3) habe ich eine Version des Fragebogens zur ideologischen Orientierung von W.F. O'Neill verwendet (Kwieciński 2012, S. 58), der für die polnische Version von Z. Melosik und P. Kwieciński angepasst worden war.

Die Untersuchung wurde im Zeitraum von Dezember 2015 bis Februar 2016 bei Studierenden der beiden Studienrichtungen ,Allgemeine Erziehungswissenschaften“ und „Sonderpädagogik“ der APS durchgeführt. Ein besonderes Augenmerk galt dem Untersuchungszeitraum direkt im Anschluss an die Wahlen zum polnischen Parlament im November 2015.

Für die Untersuchung wurde in einer mehrstufigen Zufallsauswahl eine Stichprobe gezogen. Die mehrstufige Zufallsauswahl fand in erster Linie aus organisatorischen Gründen Anwendung, die Auslosung einzelner Personen hätte die Umsetzung 
und Durchführung der Studie deutlich erschwert. Bei der Bildung des Samples habe ich versucht, für beide genannten Studienrichtungen eine repräsentative Auswahl zu treffen, weshalb Studierende aller Studienjahre von (Bachelor und Master) einbezogen wurden. Aus jedem Studienjahr wurden mindestens zwei Gruppen untersucht. ${ }^{1}$ An der Studie nahmen insgesamt 506 Personen teil, von denen wegen des hohen Anteils von Studentinnen in beiden Studienrichtungen $95 \%$ Frauen waren. Diese Dominanz weiblicher Antwortenden verschiebt, gerade auch in Bereichen wie der politischen Bildung, die Ergebnisse - Frauen sind, wie etwa die Shell-Jugendstudie in Deutschland (2015) zeigt, generell an „Politik“ weniger interessiert als Männer. In dem Bericht zu den Ergebnissen einer Repräsentativerhebung in Polen heißt es: „Multivariate Analysen lassen erkennen, dass die Kategorie Geschlecht die wichtigste Variable darstellt, die das Interesse an Politik differenziert" (CBOS 2014, S. 63). Alle Antwortenden erhielten beide Fragebogen, die adaptierte Fassung des Fragebogens von O’Neill sowie den von mir konstruierten Fragebogen. Letzterer wurde von allen (unter gelegentlicher Auslassung einzelner Antworten) ausgefüllt, ersterer ausreichend vollständig nur von 382 Studierenden. Zunächst füllten die Studierenden einen Fragebogen zur Messung ihrer ideologischen Orientierungen aus; anschließend wurden sie gebeten, den speziell erstellten Fragebogen zu beantworten.

Die Auswertung erfolgte mittels statistischer Signifikanzprüfungen. Einer ersten Häufigkeitsauszählung folgten Korrelationen insbesondere hinsichtlich Parteienpräferenz und Ansichten zur Demokratie. Die entsprechenden Hypothesen basierten auf grundsätzlichen theoretischen Überlegungen (Szafraniec 2011) und statistischer Auswertung der Wahlergebnisse zum Parlament seit 1989 (Sześciło et al. 2013; Cześnik 2007). Danach tendieren junge, in Großstädten lebende Menschen mit Hochschulausbildung dazu, für fortschrittliche, liberale und linke Gruppierungen ihre Stimmen abzugeben.

In der Zusammenführung der Daten aus den beiden Erhebungsinstrumenten zeigten sich die erwartbaren Probleme der Kompatibilität von Begriffen, Kategorien und Abstraktionsniveaus. Es erfolgte daher keine gemeinsame statistische Auswertung, jedoch eine vorsichtige vergleichende Interpretation.

\section{Ansichten zu Demokratie und Politik}

Gemeinhin wird angenommen, dass sich junge Menschen kaum für Politik interessieren. Studien in Polen verweisen auf Passivität, Misstrauen und Zurückhaltung eines erheblichen Teils von jungen Menschen der Politik gegenüber (CBOS 2015). Andere Studien hingegen in anderen europäischen Ländern dokumentieren ein starkes Engagement junger Menschen für politische Fragen. ${ }^{2}$ In unserer Studie bestätigt sich der Befund für Polen: Es ist festzustellen, dass das Interesse an Politik bei der überwiegenden Mehrheit der Befragten eher gering ist; in der Regel beschränken sie

\footnotetext{
1 Von der Untersuchung waren Fernstudenten und Doktoranden ausgenommen.

2 Vgl. https://www.jugendkultur.at/wp-content/uploads/Junge-wutbuergerinnen_grosseger_2012.pdf, Teil 2.
} 


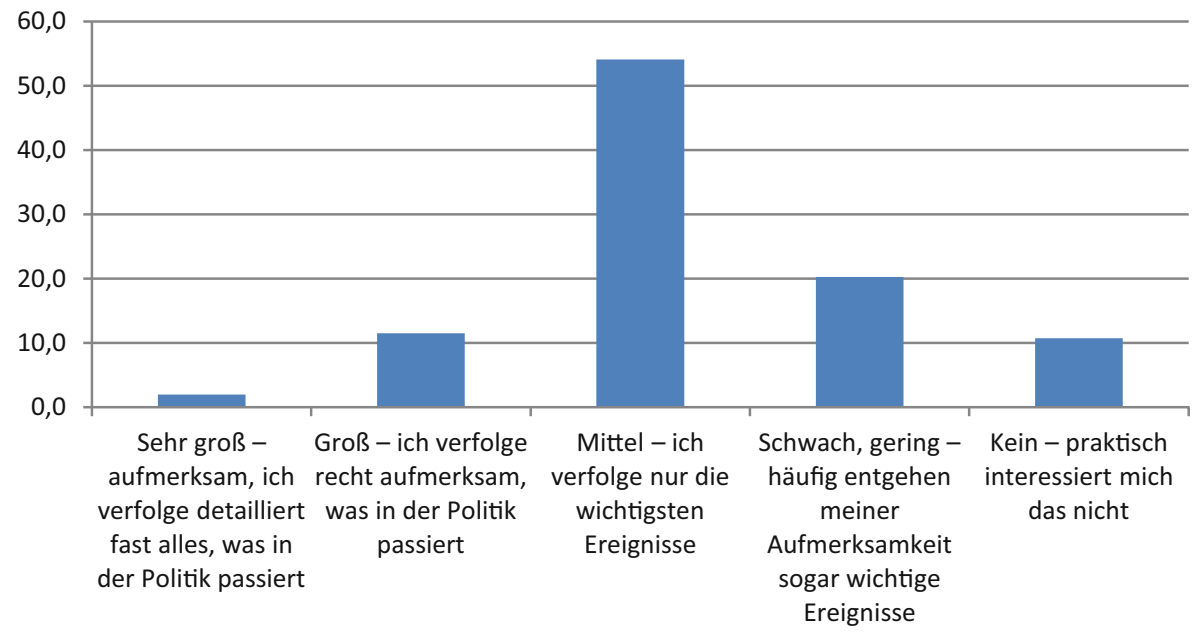

Abb. 1 Interesse an Politik. Antwort auf die Frage: Wie groß ist Dein Interesse an Politik? $(N=503$; eigene Darstellung)

sich auf das Verfolgen der wichtigsten Ereignisse (54\% der Antworten). ${ }^{3}$ Weniger als $14 \%$ der Studierenden interessieren sich in hohem oder sehr hohem Maße für Politik (davon wiederum geben nur $2 \%$ ein sehr großes Interesse an), während mehr als $30 \%$ sich überhaupt nicht für Politik interessieren (vgl. Abb. 1).

Dies bestätigt teilweise die These von einem Mangel an politischem Interesse in akademischen Kreisen in Polen. Zivilgesellschaftliche Gleichgültigkeit gilt als eine der großen Epidemien, die dort an den Universitäten grassieren. „Die Hochschulbildung ist $\mathrm{zu}$ einem Basar mit billigen Abschlüssen verkommen, die durch keinerlei Kompetenzen gedeckt sind (...). Die Ausbildung befähigt nicht zu verantwortungsvoller Bürgerbeteiligung, Wirtschaftstätigkeit und aktiver Nutzung der Kultur“ (Kwieciński 2007, S. 227). Zudem zeigen Untersuchungen auch nur geringes Interesse für öffentliche Angelegenheiten unter einem Großteil der Studierenden. ${ }^{4}$

Die Studierenden der APS sind auch nicht von der Überlegenheit der Demokratie gegenüber anderen politischen Systemen überzeugt. Der Behauptung, dass die Demokratie allen Regierungsformen überlegen ist, stimmten $39 \%$ der Befragten zu mit anderen Worten: Weniger als die Hälfte der Studierenden der APS betrachten die Demokratie als nicht verhandelbaren Wert. Etwas mehr als ein Viertel der Befragten (26\%) glaubt, dass ,man manchmal nicht-demokratischen Regierungsformen den Vorzug gegenüber demokratischen Regierungen geben sollte“. Unter den Wählerinnen und Wählern der Partei PiS ist dieser Prozentsatz nur unwesentlich höher und beläuft sich auf $29 \%$. Jede vierte Befragte akzeptiert also unter bestimmten Umstän-

\footnotetext{
3 Hier spielt die erwähnte Dominanz weiblicher Antwortender sicherlich eine große Rolle.

${ }^{4}$ Im Fragebogen habe ich jedoch nicht nach einer wirtschaftlichen, kulturellen oder sozialen Aktivität gefragt, die zum Beispiel im Zusammenhang mit der Beteiligung an karitativen Aktivitäten, Hilfsaktionen oder lokalen Initiativen zum Nutzen einer örtlichen Gemeinschaft verbunden sein können.
} 


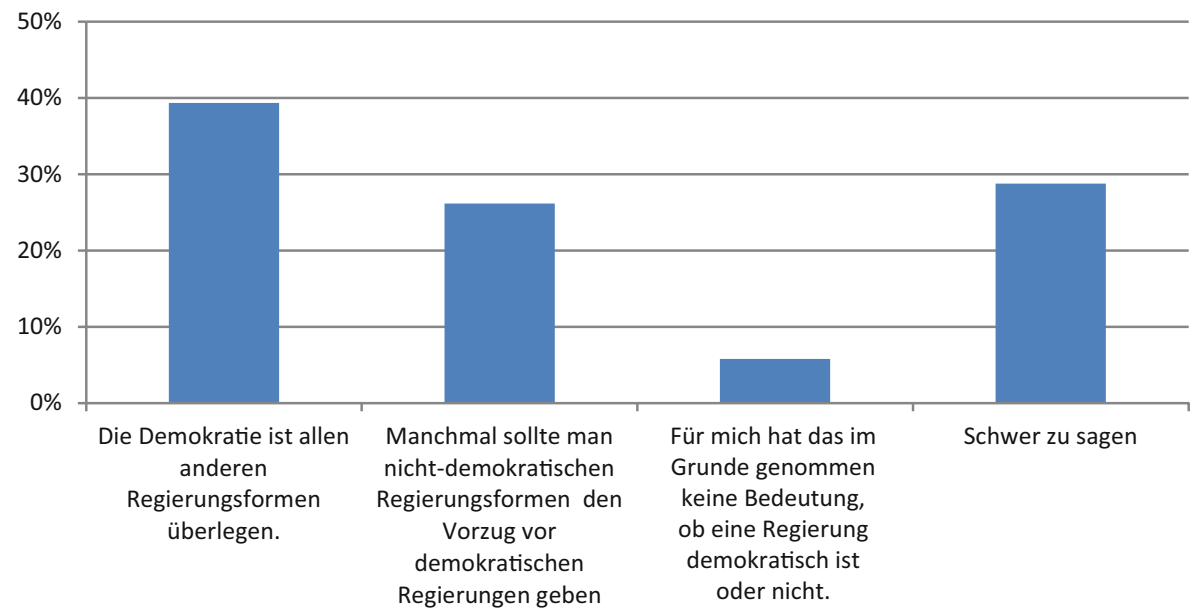

Abb. 2 Demokratie als Staatsform. Antwort auf die Frage: Welche unter den folgenden Aussagen kommt Deinen Überzeugungen am nächsten? $(N=501$; eigene Darstellung)

den die Einführung eines anderen politischen Regimes. Nur 6\% sind der Ansicht, dass das politische System keine Bedeutung spielt. Allerdings wählten beinahe $30 \%$ der Befragten die Antwort „schwer zu sagen“, was ein Hinweis darauf sein kann, dass sie die Art des politischen Systems überhaupt nicht interessiert oder dass sie nicht über ausreichende Kenntnisse verfügen, um hierzu ein Urteil zu haben (vgl. Abb. 2).

Darüber hinaus wurden die Studierenden gebeten, ihre politischen Ansichten auf einer Skala von 1 bis 10 zu definieren, wobei „1“ für eine linke und „10“ für eine rechte Gesinnung stehen. Die größte Gruppe unter den Befragten (36\%) wählte die mittlere Antwort (5). Der Durchschnitt aller Antworten liegt bei 5,88. Unter denjenigen, die ihre Unterstützung für die Regierungspartei PiS erklärten, liegt die durchschnittliche Antwort mit 7,66 signifikant ${ }^{5}$ höher. Extreme Antworten sind häufiger auf der rechten Seite der Achse zu finden. Die „10“ wurde von $5 \%$ der Befragten gewählt, demgegenüber entschieden sich nur $2 \%$ für die „1“ (vgl. Abb. 3).

Die Verteilung der Antworten führt zur folgenden Schlussfolgerung: Die überwiegende Mehrheit der Befragten (2/3) ist der politischen Mitte zuzurechnen; über $20 \%$ sind erklärte Anhänger der Rechten, hingegen nur $10 \%$ sind als Linke einzuschätzen. ${ }^{6}$

Es besteht eine Abhängigkeit zwischen dem Maß des Interesses an Politik und den angegebenen Ansichten, sie ist statistisch signifikant (bei Signifikanzniveau 0,05;

\footnotetext{
5 Es besteht eine statistisch signifikante Abhängigkeit. Die Abhängigkeit zwischen den Gruppen wurde verglichen anhand des ANOVA Tests und des Eta-Koeffizienten $(\mathrm{p}<0,05$; Eta-Quadrat $=0,185)$.

6 Die Ansichten der Befragten habe ich in drei Gruppen unterteilt: links, mitteorientiert und rechts. Die Methodik für die Bestimmung der Ansichten der Befragten stützte sich auf die Klassifizierung konkreter Antworten auf eine der drei als konventionell angenommenen Strömungen. Daraus folgt: Als Anhänger der Linken habe ich Personen qualifiziert, die Antworten im Bereich 1-3 markierten; bei Anhängern der politischen Mitte ging ich davon aus, dass sie die Antworten 4, 5, 6 oder 7 wählen würden; zu den Anhängern rechter Ansichten rechnete ich jene Personen, die auf der Achse die Antworten 8, 9 oder 10 markierten.
} 


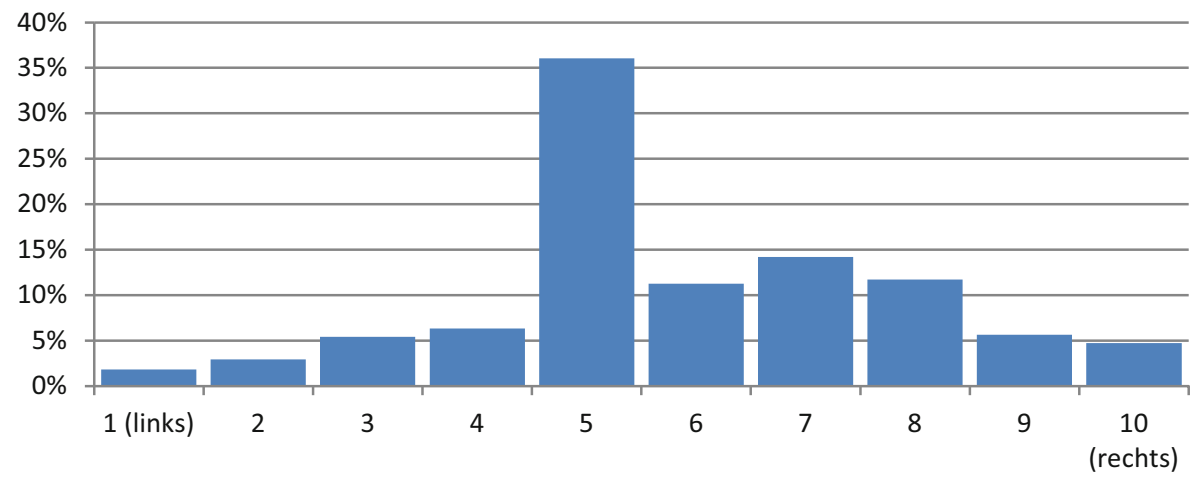

Abb. 3 Politische Einstellung. Prozentuale Verteilung der erklärten politischen Ansichten der Studierenden auf einer Skala von 1 bis 10, wobei 1 für eine linke und 10 für eine rechte Gesinnung steht $(N=444$; eigene Darstellung)

$\chi 2=33,698, p=0,00000085954)$. In allen Gruppen dominieren Anhängerinnen und Anhänger der politischen Mitte. Unter den Personen mit hohem oder sehr hohem Interesse an Politik (nur $14 \%$ der Studierenden) dominieren ebenfalls die ,Zentristen“, jedoch deutlich schwächer als in den anderen Gruppen. Unter den Befragten, die sich für Politik interessierten, gehören $45 \%$ der politischen Mitte an, wohingegen mehr als $40 \%$ rechte Ansichten vertreten. Unter den Personen, die sich in mindestens hohem Ausmaß für Politik interessieren, ist ein deutliches Übergewicht derjenigen mit rechtsgerichteten Ansichten gegenüber solchen mit linken Überzeugungen festzustellen (die in dieser Gruppe nur $14 \%$ ausmacht). Unter denjenigen, die kaum Interesse an Politik zeigen, befindet sich der Prozentsatz der Anhänger der politischen Mitte auf einem viel höheren Niveau. Unter den Studierenden, die ein geringes oder gar kein Interesse an der Politik bekunden (fast ein Drittel aller Befragten), erklären sich $85 \%$ zu Angehörigen der ,,politischen Mitte“ (hierbei kann davon ausgegangen werden, dass diese Menschen vorsichtshalber Antworten auf der Mitte der Skala bevorzugen).

Etwa die Hälfte $(50,4 \%)$ der Befragten verwies auf eine bestimmte Partei, für die sie gestimmt haben. Andererseits gaben $37 \%$ der Befragten an, dass sie nicht an den Wahlen teilgenommen haben. Die restlichen $12 \%$ weigerten sich, diese Frage zu beantworten oder konnten sich nicht erinnern, für wen sie gestimmt hatten (diese Gruppe der Befragten habe ich den „Nicht-Wählern“ hinzugerechnet). Die Wahlbeteiligung unter den Studierenden war gering - sie lag um 0,5 Prozentpunkte unter dem offiziellen Wert (50,92\%). Zudem sollte davon ausgegangenen werden, dass sich der tatsächliche Prozentanteil der an den Wahlen teilnehmenden Studierenden auf einem noch niedrigeren Niveau befand, als dies für die Zwecke der Untersuchung von den Befragten erklärt wurde.

Die Partei PiS erhielt bei den Wahlen 37,6\%, was ihr den Gewinn von 235 Mandaten im 460 Sitze umfassenden Sejm erlaubte. Der Anteil derjenigen Studierenden der Hochschule, die im Fragebogen ihre Unterstützung für die Regierungspartei erklärten, betrug $36 \%$. Dieses Ergebnis unter den Studierenden mag überraschend sein, da die PiS traditionell eher bei Menschen aus kleineren Städten und mit einem 
niedrigeren Bildungsniveau die größte Anhängerschaft hat. Man könnte meinen, dass die großstädtische Jugend (oder zumindest in einer Großstadt lebende junge Menschen) eher liberale und linke Ansichten teilen. Die Unterstützung für die PiS unter den Pädagogik-Studierenden liegt damit höher als durchschnittliche Ergebnisse bei den jüngeren Altersgruppen.

\section{Einschulung, Schulstruktur und Religion}

Die Partei PiS trat mit zwei Bildungsthemen an, um die herum - während der gesamten Dauer der Wahlkampagne, aber auch noch in der Regierungszeit - Auseinandersetzungen geführt wurden. Die vorherige Regierungsmannschaft unter Premierminister Donald Tusk hatte das Schulpflichtalter von sieben auf sechs Jahre abgesenkt. Eine landesweite Kampagne der Befürworter einer erneuten Erhöhung des Schuleintrittsalters, die durch PiS unterstützt wurde, fand unter dem Motto „Rettet unsere Kleinen“" statt. Es wurde argumentiert, dass die Schulen nicht auf die Aufnahme der Sechsjährigen vorbereitet seien, dass hier ein Mangel an Freiluft-Aktivitäten zu beklagen sei und die Schule die Kräfte eines sechsjährigen Kindes übersteige. Auf der anderen Seite verwiesen viele Verteidiger der „Schule ab sechs“ auf Trends in der EU oder auf die Notwendigkeit, Schulen auf die Herausforderungen der modernen Welt hin anzupassen.

Die Studierenden der APS nahmen die Initiative von PiS zur Erhöhung des Schuleintrittsalters mit Begeisterung auf: Zwei Drittel der Befragten unterstützten sie. Unter den Anhängerinnen und Anhängern von PiS betrug dieser Anteil über $80 \%$. Ähnliche Ergebnisse wurden im Übrigen von landesweiten repräsentativen Umfragen (IBRiS 2015) ermittelt. Eine Mehrheit der befragten Studierenden (67\%) war der Ansicht, dass die Entscheidung über das Schuleintrittsalter auf dem Wege eines Referendums entschieden werden sollte. Gleich zu Beginn der Wahlperiode verabschiedete der Sejm eine Novellierung des Schulgesetzes, das wie bereits zuvor Kinder erst ab dem sibten Lebensjahr für die Schulpflicht erfasst.

Die zweite wichtige Bildungsposition der PiS-Wahlkampagne traf auf heftigen Widerstand vor allem aus dem Umfeld von Lehrern und Pädagogen. Seit dem Jahre 1999 bestand das polnische Bildungssystem aus einer sechsjährigen Grundschule, dem dreijährigen Gymnasium und dem dreijährigen Lyzeum (bzw. aus einer dreijährigen Berufsschule oder einem vierjährigen Technikum). PiS kündigte die Beseitigung des Gymnasiums und die Rückkehr zu einem System aus achtjähriger Grundschule und vierjährigem Lyzeum an, so wie das zuvor der Fall gewesen war. Als Hauptargument wurden vor allem Erziehungsprobleme in den Gymnasien angeführt, die ein Hort der Aggression und Gewalt seien. PiS versicherte den Gymnasiallehrerinnen und -lehrern zugleich, dass alle in den neuen Lyzeen Arbeit finden würden. Allerdings glaubten diese den Versprechungen nicht und gaben dem wiederholt in Protesten und öffentlichen Reden Ausdruck. Der Verband der Polnischen Lehrerschaft führte im November 2015 eine Umfrage unter mehr als 71.000 Gymnasiallehrerinnen und -lehrern durch, die gezeigt hat, dass $84 \%$ von ihnen den Verlust ihres Arbeitsplatzes wegen nicht ausreichender Stundenzahlen an den neu organisierten Schulen befürchteten (ZNP 2016). Die Gegner des Regierungsprojekts 
betonten auch, dass die Abschaffung der Gymnasien keineswegs die Mängel des polnischen Schulsystems beseitigen werde; die Verlegung der Schülerinnen und Schüler aus einer Schule in eine andere sei für sich genommen noch kein Beitrag zur Lösung von Problemen. Zudem verwiesen sie auf das Ausbleiben einer echten, eingehenden und inhaltlichen Analyse zu den Folgen der Veränderungen im Schulsystem.

In der Untersuchung habe ich die Studierenden über ihre Einstellung zur Beseitigung der Gymnasien und zur Wiedereinführung der achtjährigen Grundschule befragt. Fast die Hälfte (48 \%) der künftigen Pädagoginnen und Pädagogen akzeptierte diese Idee (unter PiS-Anhängern betrug dieser Prozentsatz 56\%, also nicht signifikant mehr), ablehnend reagierten $35 \%$ der Befragten. Eine Mehrheit der Studierenden $(60 \%)$ vertritt hingegen die Ansicht, dass die Frage der Beseitigung des Gymnasiums in einem Referendum entschieden werden sollte.

Die Ergebnisse zeigen, dass die Meinungen unter den Studierenden der APS in dieser Sache weit auseinandergingen. Es sollte betont werden, dass die Untersuchung zur Jahreswende 2015/2016 durchgeführt wurde. Repräsentative landesweite Umfragen (IBRiS 2015) aus demselben Zeitraum zeigten eine noch stärkere Unterstützung für die Idee der Abschaffung der Gymnasien (fast $60 \%$ waren mit der vorgeschlagenen Abschaffung der Gymnasien einverstanden).

PiS hat zum Ende des Jahres 2016 per Gesetz das dreijährige Gymnasium abgeschafft und den Zustand von vor 1999 mit der achtjährigen Grundschule und dem vierjährigen Lyzeum wiederhergestellt. Die Veränderungen im Schulsystem gehen in der Folge einher mit einer Änderung der Lehrpläne, die mit der Einführung neuer Leselisten und neuer Lerninhalte verbunden ist. Es mehren sich die Anzeichen dafür, dass die Korrektur der Struktur des Bildungssystems sich auch in einer Revision der Curricula im konservativ-nationalen Geiste widerspiegeln wird. Das Wahlprogramm der PiS sah den schwerwiegendsten Fehler des Schulsystems in inhaltlichen und ideologischen Defiziten; es kann daher erwartet werden, dass mit Nachdruck eine sogenannte patriotische und religiöse Erziehung forciert werden wird.

Eine Frage, die seit vielen Jahren kontrovers diskutiert wird, ist der Religionsunterricht in den Schulen. Boguslaw Śliwerski (2015, S. 198) stellt fest: „Im Zusammenhang mit dem Engagement staatlicher Behörden Polens bei der Förderung oder Verdrängung der Religion (je nachdem, welche politische Partei gerade an der Macht ist) erweist sich das Verhältnis zwischen staatsbürgerschaftlicher Erziehung und Religion als ein besonderes Problem." In jeder Wahlkampagne bieten Fragen der Anwesenheit von religiösen Symbolen in den Schulen, der Religionsunterricht und seine Finanzierung sowie die Benotung und deren Einbezug in die Durchschnittsnote ein breites Feld für ideologische Auseinandersetzungen. Nach 1989 wurde der Religionsunterricht in den Schulen eingeführt. Die Teilnahme an ihm ist hingegen freiwillig, je nach den Wünschen der Eltern oder Schülerinnen und Schüler. Seit dem Schuljahr 2007/2008 wird er in die Berechnung des Notendurchschnitts einbezogen, hat allerdings keinen Einfluss auf die Versetzung in die nächste Klasse.

Die überwiegende Mehrheit der Studierenden der APS akzeptiert hierbei den Status quo. Mehr als $60 \%$ sind der Meinung, dass Religionsunterricht an den Schulen fakultativen Charakter haben sollte. Jeder fünfte Befragte spricht sich hingegen für eine allgemeine Teilnahmepflicht aus. Im Gegensatz dazu vertreten nur $13 \%$ die Ansicht, dass der Religionsunterricht in der Schule nichts zu suchen habe. Die 
überwiegende Mehrheit der Befragten ist überdies der Ansicht, dass katholische Religionssymbole in der Schule anwesend sein sollten (37\%) oder können (35\%). $15 \%$ ist diese Frage ganz gleichgültig. Weniger als einer von zehn Studierenden ist der Meinung, dass für religiöse Symbole kein Platz in öffentlichen Schulen sei. Zum Vergleich: Nur $30 \%$ stimmen der Darstellung von Symbolen anderer Religionen in Schulen zu. Fast $65 \%$ der Befragten lehnen den Einbezug des Regionsunterrichts in den Notendurchschnitt ab, nur $20 \%$ stimmen hier zu. Zur gleichen Zeit hält fast die Hälfte der Befragten an der These fest, dass der Religionsunterricht aus Steuermitteln finanziert werden sollte. Jeder vierte Befragte ist der Meinung, dass die Kosten für den Religionsunterricht von den Glaubensgemeinschaften getragen werden sollten, $6 \%$ sprechen sich hingegen dafür aus, die Eltern jener Kinder, die an solchen Aktivitäten teilnehmen möchten, mit den entsprechenden Kosten zu belasten.

Im Fragebogen habe ich die Studierenden auch darum gebeten, sich zu verschiedenen Ideen zu äußern, die in den Wahlprogrammen anderer, ebenfalls für das Parlament kandidierender Parteien enthalten sind. Einige von ihnen wiederholten sich in den Wahlprogrammen der meisten Gruppierungen. Dazu zählte zum Beispiel die Position, dass die ,Vorschulerziehung gleichermaßen zugänglich und kostenlos wie die Grundschulbildung sein sollte" und dass eine Notwendigkeit für die medizinische und zahnmedizinische Versorgung in Schulen und Kindergärten bestehe. Dieser Aussage stimmten mehr als $90 \%$ der Studierenden zu. Kaum geringer war die Unterstützung der Befragten für die Forderung nach ,kostenlosem Frühstück und Mittagessen für jedes Kind im Kindergarten und in der Schule“ (85\%) sowie zur „Einführung kostenloser Lehrbücher in Grund- und Mittelschulen“ (83\%). Bei diesen Vorschlägen handelt es sich jedoch um überparteiliche Positionen. Seit vielen Jahren macht so ziemlich jede Partei ähnliche Versprechungen. Wie daran ersichtlich ist, treffen Forderungen nach dem Ausbau wohlfahrtsstaatlicher Funktionen auf die Zustimmung einer großen Mehrheit der Befragten. ${ }^{7}$

\section{Bildungsorientierungen}

Der Fragebogen von W.F. O’Neill erfasst sechs Bildungsideologien, die auf 14 Aussagen beruhen, die abgefragt werden. Unterschieden wird in der Aggregation zwischen: Fundamentalismus (F), Intellektualismus (I), Bildungskonservatismus (Ke), Bildungsliberalismus (Le), Emanzipationsideologie (E) und Bildungsanarchismus (A). ${ }^{8}$

\footnotetext{
7 Die geringste Wertschätzung der Befragten erfuhren Forderungen der ultraliberalen Partei KORWIN, die von dem bekannten Exzentriker und Mitglied des Europäischen Parlaments Janusz Korwin Mikke geführt wird. Unter den Studierenden kam die Partei auf beinahe $10 \%$ (bei den Wahlen konnte sie die $5 \%$ Hürde nicht überspringen und verpasste so den Einzug ins Parlament). Die beiden im Programm dieser Partei enthaltenen Vorschläge - „Der polnische Staat sollte sich aus der Durchführung und Finanzierung von Maßnahmen im Bildungsbereich zurückziehen“ und „Die Schulpflicht muss abgeschafft werden“ erfuhren nur marginale Unterstützung der Studierenden ( $8 \%$ bzw. $5 \%$ ).

8 Der Fragebogen enthält 104 Behauptungen in Bezug auf die Bildungsphilosophie und die Vision von Schule, dabei insbesondere solche Aspekte, die die Bildungsideologie formen, wie z. B. das allgemeine Bildungsziel, Aufgaben und Funktionen der Schule, Verwaltung und Kontrolle des Schulsystems, Charakter der Lehrpläne, Lerninhalte sowie Lernmethoden und Beurteilung (O’Neill 1981, S. 21). Auf jede der
} 
Tab. 1 Bevorzugte Bildungsideologien. Dominanz der Indikationen für einen bestimmten Typ von Bildungsideologie unter Einbezug der Studienrichtung ( $N=382$; eigene Darstellung)

\begin{tabular}{|c|c|c|c|c|c|c|}
\hline $\begin{array}{l}\text { Bildungs- } \\
\text { ideologien }\end{array}$ & $\begin{array}{l}\text { Fundamenta- } \\
\text { lismus }\end{array}$ & $\begin{array}{l}\text { Intellektua- } \\
\text { lismus }\end{array}$ & $\begin{array}{l}\text { Bildungskon- } \\
\text { servatismus }\end{array}$ & $\begin{array}{l}\text { Bildungslibe- } \\
\text { ralismus }\end{array}$ & $\begin{array}{l}\text { Emanzipations- } \\
\text { ideologie }\end{array}$ & $\begin{array}{l}\text { Bildungsanar- } \\
\text { chismus }\end{array}$ \\
\hline Gesamt (\%) & 4 & 3 & 6 & 67 & 11 & 8 \\
\hline
\end{tabular}

Unter den Studierenden der APS dominieren ganz eindeutig Personen mit liberalen Ansichten zur Bildung. Die liberale Erziehung wird sichtbar am stärksten von den Studierenden respektiert. Auffällig ist auch der relativ hohe Anteil von Personen mit linken Ansichten. Unter fast $20 \%$ der Studierenden dominiert eine liberale (Emanzipationsideologie: $11 \%$ ) oder anarchistische Orientierung (Bildungsanarchismus: $8 \%$ ) (vgl. Tab. 1). Wie zu sehen ist, sind die drei beliebtesten Bildungsideologien der liberalen oder linken Strömung zuzurechnen. Demgegenüber kommen die streng Konservativen („Fundamentalismus“ und „Intellektualismus“) nur auf einen Wert von $7 \%$ der Befragten. Die Unterstützung für den Bildungskonservatismus liegt auf einem niedrigeren Niveau als jede einzelne der progressiven Bildungsideologien. Die Studierenden beider Studienrichtungen sind, ganz unabhängig vom Jahrgang, Anhänger von liberalen und linken Bildungsideologien. Damit kann getrost davon ausgegangen werden, dass rechtsextreme Orientierungen unter den Studierenden der APS nur in ganz geringem Maße auftreten.

Jede der Skalen der einzelnen Bildungsideologien bestand, wie erwähnt, aus 14 Aussagen. Die mit Abstand höchsten Durchschnittswerte $(11,94)$ sind auf der Skala des Bildungsliberalismus zu beobachten. Auf dieser Skala finden sich auch die bei Weitem höchsten Mediane und Dominanten. Die niedrigsten Durchschnittswerte entfallen auf den „Fundamentalismus“ und „Intellektualismus“. In den Aussagen zur Skala F gingen die Meinungen der Untersuchten auch am stärksten auseinander $(\mathrm{SD}=6,911)$. Die kleinste Varianz lässt sich wiederum auf der Skala E $(\mathrm{SD}=5,197)$ und Le $(\mathrm{SD}=5,335)$ beobachten (vgl. Tab. 2).

Die Verteilung der Ergebnisse legt den Verdacht nahe, dass es sich hier entweder um eine weltanschauliche Verwirrung oder den Mangel an präzisen Ansichten der Befragten in Bildungsfragen handelt. Auf der einen Seite nähern sich die Befragten spezifischen Fragen in den einzelnen Bildungsideologien auf vielfältige Weise. Beispielsweise stimmen sie den Zielen der Konservativen zu, bevorzugen dabei aber gleichzeitig liberale Inhalte und Lehrmethoden. Auf der anderen Seite hingegen zeigt die Verteilung der Antworten, dass die Befragten eher zur Zustimmung als zur Ablehnung von einzelnen Aussagen neigen. Mehr als die Hälfte der Antworten $(54 \%)$ in dem Fragebogen entfallen auf „Stimme zu“ oder „Stimme völlig zu“

Behauptungen konnten die Teilnehmer und Teilnehmerinnen mithilfe einer Fünf-Punkte-Skala reagieren, wobei ,,-2“ „Ich bin überhaupt nicht einverstanden“; ,-1“ „Stimme nicht zu“; ,0“ „Ich bin unsicher, unentschieden oder indifferent“; ,,+1“ „Stimme zu“; und ,,+2“ „Stimme völlig zu“ bedeuteten. Die Skala der Bildungsideologien wurde aus 14 Aussagen gebildet, weswegen ein Teilnehmer mindestens einen Wert von minus 28 erreichen konnte, wohingegen der Höchstwert bei plus 28 Punkten lag. Jedem Befragten wurde auf der Grundlage der erteilten Antworten auf die im Text enthaltenen Aussagen eine dominierende Ideologie zugeschrieben, also jene Ideologie, hinsichtlich derer er die höchste Punktzahl erhalten hatte. Die Ergebnisse auf einer bestimmten Skala wurden durch Summierung der Punkte berechnet. Auf diese Weise war es möglich, die Dominanz einer bestimmten Weltsicht gegenüber anderen zu bestimmen. 
Tab. 2 Bildungsideologien differenziert (eigene Darstellung)

\begin{tabular}{lllllllll}
\hline & $\begin{array}{l}\text { Durch- } \\
\text { schnitt }(\mathrm{M})\end{array}$ & $\begin{array}{l}\text { Median } \\
(\mathrm{Me})\end{array}$ & $\begin{array}{l}\text { Dominante SD } \\
(\mathrm{Mo})\end{array}$ & Varianz & $\begin{array}{l}\text { Beobachteter } \\
\text { Mindestwert }\end{array}$ & $\begin{array}{l}\text { Beobachteter } \\
\text { Höchstwert }\end{array}$ & $N$ \\
\hline $\begin{array}{l}\text { Fundamenta- } \\
\text { lismus }\end{array}$ & 2,95 & 3 & 3 & 6,911 & 47,76 & -23 & 19 & 483 \\
$\begin{array}{l}\text { Intellektua- } \\
\text { lismus }\end{array}$ & 4,11 & 4 & 4 & 5,549 & 30,79 & -13 & 20 & 488 \\
$\begin{array}{l}\text { Bildungskon- } \\
\text { servatismus }\end{array}$ & 6,36 & 7 & 5 & 6,040 & 36,48 & -14 & 24 & 484 \\
$\begin{array}{l}\text { Bildungslibe- } \\
\text { ralismus }\end{array}$ & 11,94 & 12 & 13 & 5,335 & 28,47 & -7 & 28 & 481 \\
$\begin{array}{l}\text { Emanzipations- } 8,07 \\
\text { ideologie }\end{array}$ & 8 & $5,00^{\mathrm{a}}$ & 5,197 & 28,24 & -15 & 22 & 489 \\
$\begin{array}{l}\text { Bildungsanar- } \\
\text { chismus }\end{array}$ & 5,48 & 5 & $7,00^{\mathrm{a}}$ & 6,126 & 37,53 & -11 & 25 & 477 \\
\hline
\end{tabular}

${ }^{\mathrm{a} E s}$ existieren viele Modi. Sichtbar ist der niedrigste Wert

(18\%). Demgegenüber waren zusammengenommen nur $20 \%$ aller Antworten negativer Natur: „Ich bin überhaupt nicht einverstanden“ (5\%) und „Stimme nicht zu“ (15\%). Die Befragten befürworteten also häufiger Aussagen, anstatt sie abzulehnen.

Die durchgeführte Untersuchung hatte im Zusammenhang mit einer der vielen bestehenden akademischen Klassifizierungen für Ideologien Sondierungscharakter und war diagnostischer Natur. Die Verteilung der Typen der Bildungsideologien verweist auf eine Dominanz des Liberalismus unter den Studierenden der Akademie für Sonderpädagogik. Auf der einen Seite sind diese Ergebnisse nicht überraschend. In den vergangenen Jahrzehnten hat sich Polen einen festen Platz unter jenen demokratischen Staaten erworben, in denen liberale und progressive Ansichten hinsichtlich des Funktionierens des Staatswesens einschließlich Bildung und Schulwesen vorherrschen. Daher ist es verständlich und natürlich, dass aus dem Westen ins Land kommende Ideen hier auch ihre Anhänger finden; sie werden immer mehr akzeptiert und stark verinnerlicht, vor allem von, wie man annehmen kann, offeneren und besser ausgebildeten Studierenden.

Doch auf der anderen Seite gibt die Dominanz der liberalen Richtung bei vernachlässigbarer Unterstützung für rechtsradikale Ansichten zur Bildung durchaus zu denken in einer Zeit, in der verschiedene Untersuchungen der Jugendforschung sowie Beobachtungen des öffentlichen Lebens zeigen, dass ein Teil der Jugendlichen unter den Einfluss rechtsradikaler Kreise gerät, die entschieden illiberale Lösungen vertreten. Das Ergebnis der Untersuchung könnte durch den Ort, an dem sie durchgeführt wurde, beeinflusst worden sein. Die Akademie für Sonderpädagogik zählt zu den angesehensten pädagogischen Hochschulen. Auch die gewählte Studienpopulation - Studierende der Pädagogik - könnte sich in ihren Ansichten stark von der Jugend im Allgemeinen unterscheiden, die - wie andere Studien zeigen (CBOS 2015) - zunehmend konservative Ansichten vertritt. 


\section{Schlussfolgerungen}

Die Veränderungen im Bildungssystem rufen in Polen keine solche Resonanz hervor, wie das zum Beispiel der Fall ist bei Fragen der Pressefreiheit oder der Versuche, die Justiz der Exekutive zu unterstellen. Bisher haben die vorgeschlagenen Lösungen in erster Linie strukturellen Charakter (Änderung des Schuleintrittsalters und Abschaffung der Gymnasien). In diesem Zusammenhang wird es aber absehbar auch zu einer radikalen Veränderung der Lehrbücher, Lehrinhalte und Bildungsziele kommen.

Die Ergebnisse der an der Akademie für Sonderpädagogik durchgeführten Untersuchung zeigen eine starke Unterstützung der jungen Erwachsenen für die siegreich aus den Wahlen hervorgegangene Partei und ihre Ideen. Die Partei selbst bezeichnet sich als konservativ. Ihre bildungspolitischen Leitlinien müssen ebenfalls als klar konservativ eingestuft werden. Andererseits ergibt sich im Lichte der Untersuchung der Bildungsideologie ein anderer Eindruck vom Weltbild der Studierenden: Es dominieren hier die Anhänger der liberalen Bildungsideologie (vgl. Tab. 1 und 2). Diese Diskrepanz kann in einer mangelnden Kenntnis der politischen Programme der an den Wahlen teilnehmenden politischen Parteien begründet liegen - die Studierenden erklärten im Rahmen der Untersuchung, dass sie sich für Politik eher nicht interessieren.

Die große Unterstützung für die Regierungspartei sei zudem weniger auf die Identifikation mit der PiS zurückzuführen, sondern könnte ein Ausdruck für die Abneigung gegenüber den während der letzten beiden Wahlperioden den Ton angebenden politischen Eliten sein. Bis zu einem gewissen Grad kann ein Grund auch in der Sympathie für eine starke, klar erkennbare Führungspersönlichkeit liegen, die von katholischen Kreisen und der Kirche unterstützt wird - im Falle des konservativen Polen kann man das nicht auszuschließen. Zudem scheint es - paradoxerweise - so zu sein, dass die Bildungspolitik kein besonderes Interessengebiet von Pädagogik-Studierenden ist. Ihre Aufmerksamkeit wird in einem größeren Ausmaß von den Positionen der politischen Parteien hinsichtlich der Aufnahme von Flüchtlingen, der Legalisierung homosexueller Beziehungen oder der Verwendung der In-vitroMethode angezogen - um nur einige der kontroversesten und spektakulärsten Auseinandersetzungen der vergangenen Monate zu nennen. Es ist ersichtlich, dass sich die Studierenden bevorzugt als Anhänger rechtsgerichteten Gedankenguts erklären. Die Frage lautet demgemäß: Stimmen sie für die PiS, weil sie rechte Überzeugungen haben, oder ist es vielleicht gerade umgekehrt und sie bezeichnen sich selbst als Menschen mit rechten Ansichten, weil sie eine Partei unterstützen, die von sich selbst als „Vereinigte Rechte“ spricht?

Die Untersuchungsergebnisse belegen überdies das allgemein geringe Interesse der Pädagogik-Studierenden an öffentlichen Angelegenheiten und ihre geringe Beteiligung an jeder Form von politischer Aktivität. Leider fehlt ihnen auch die Überzeugung von der Überlegenheit demokratischer Systeme gegenüber anderen. Mit Blick darauf entsteht eine weitere, vielleicht sogar die wichtigste Frage: Was verstehen junge Polen unter einem demokratischen System, worauf beruht ihrer Meinung nach dessen Wesen? Die Beobachtung des gesellschaftlichen Lebens verleitet zur Formulierung der These, dass wir alle eine Niederlage erlitten haben: Die Demokratie wird von der Mehrheit als Ausdruck des Mehrheitswillens betrachtet, 
der alle Handlungen und Entscheidungen rechtfertigen soll. Für die konzeptionelle Arbeit in der politischen Erwachsenenbildung ergeben sich mehrere Konsequenzen aus diesen Befunden.

Zum Ersten scheint es so zu sein, dass die Lernziele nicht nur auf Demokratie, gesellschaftliche Teilhabe und Formen politischer Willensbildung gerichtet sein können, da dies auf der ,ideologischen“ Ebene bleibt und wenig Wirkung auf konkrete Einstellungen und Verhaltensweisen hat. Sie müssen sich den Diskrepanzstrukturen zuwenden, welche zwischen abstrakten Ideologien und konkreten, kontextabhängigen politischen Entscheidungen bestehen. Dies ist nicht nur ein kognitiver Prozess, sondern ein Lernprozess, der kulturelle Traditionen, Ängste, Glauben und konkrete aktuelle Interessen gleichermaßen erfasst. Allgemeine demokratietheoretische Positionen müssen, wenn man so will, im Prozess der politischen Erwachsenenbildung auch didaktisch mit konkreten Handlungsbedarfen und Optionen verbunden werden.

Zum Zweiten gibt es für das Bildungswesen insgesamt sehr zu denken, dass es ausgerechnet die künftigen Lehrkräfte in Schulen und anderen Bildungseinrichtungen sind, die in einer inkohärenten und widersprüchlichen Weise Alltagspolitik und demokratiebezogene Bildungspositionen verbinden. Es wäre also nicht nur an eine Weiterentwicklung der politischen Erwachsenenbildung zu denken, sondern auch an eine verstärkte Implementation politischer Bildung in den Hochschulen, an denen zukünftige Lehrende ausgebildet werden.

Zum Dritten ergeben sich deutlich Forschungsbedarfe zu den komplexen und widersprüchlichen Ausgangspunkten politischer Bildung bei den Menschen, die mit großer gesellschaftlicher Relevanz an der Schnittstelle von Demokratie und Bildung arbeiten. Hier sind Kausalitäten und Zusammenhänge zu überprüfen, empirische Grundlagen für didaktische Konzepte zu entwickeln und Wirkungen zu evaluieren.

Open Access Dieser Artikel wird unter der Creative Commons Namensnennung 4.0 International Lizenz (http://creativecommons.org/licenses/by/4.0/deed.de) veröffentlicht, welche die Nutzung, Vervielfältigung, Bearbeitung, Verbreitung und Wiedergabe in jeglichem Medium und Format erlaubt, sofern Sie den/die ursprünglichen Autor(en) und die Quelle ordnungsgemäß nennen, einen Link zur Creative Commons Lizenz beifügen und angeben, ob Änderungen vorgenommen wurden.

\section{Literatur}

Ahlheim, K., \& Mathes, H. (2005). Plädoyer für eine kritische politische Bildung. http://www.linksnet.de/ artikel.php?id=1647. Zugegriffen: 22. Jan. 2008.

BMI - Bundesministerium des Inneren (2001). Erlass über die Bundeszentrale für politische Bildung vom 24. Januar 2001. Berlin: BMI.

Brandt, P., Rudolf, K., \& Länge, T. (2003). Junger Wilder trifft alten Hasen: „Bericht politische Bildung 2002“. DIE Zeitschrift für Erwachsenenbildung, 2, 24-28.

CBOS - Centrum Badania Opinii Społecznej (2014). Młodzież 2013. Warszawa: CBOS.

CBOS - Centrum Badania Opinii Społecznej (2015). Komunikat z badań Nr 135. Zainteresowanie politykq i poglady polityczne $w$ latach 1989-2015. Deklaracje ludzi młodych na tle ogótu badanych. Warszawa: CBOS.

Cześnik, M. (2007). Partycypacja wyborcza w Polsce: perspektywa porównawcza. Warszawa: Wydawnictwo Naukowe „Scholar“.

The Economist (2016). Democracy Index 2016, Revenge of the „,deplorables“. A report by the Economist IBRIS - Instytut Badań Społecznych i Rynkowych (2015). Społeczna ocena propozycji zmian systemu edukacji. Warszawa. http://www.ibris.pl/Spoleczna_ocena_propozycji_zmian_systemu_edukacji. Zugegriffen: 1. Febr. 2016. 
Intelligence Unit (2016). Intelligence Unit. http://pages.eiu.com/rs/783-XMC-194/images/Democracy_ Index_2016.pdf?mkt_tok=eyJpIjoiTVdFM05HUXpaVEU0Wm1NMyIsInQiOiIyQ1wvVHRLaUww SF10dW9LMk1NZ0loWmY0cmk0dkYxWHZZRCt3WXZWaEJGemRrUTh5bTJHWExJc2IwcGRq eE1uRVwvWXpDbEtLRkVSRUYzbFRCbnNQVG9peDRqdU03MXpwNmJkT281NTlqaWh3V0dn ZFJXWDZsMmdWR0puVEhpRHNvIn0\%3D. Zugegriffen: 16. Jan. 2016.

Kwieciński, Z. (2007). Między patosem a dekadencja. Studia i szkice z socjopedagogiczne. Wrocław: Wydawnictwo Naukowe Dolnośląskiej Wyższej Szkoły Edukacji TWP.

Kwieciński, Z. (2012). Pedagogie postu. Preteksty. Konteksty. Podteksty. Kraków: Oficyna Wydawnicza „Impuls".

Michnik, A. (im Gesprach mit) Wielowieyska, D. (18.05.2016). Zobaczyłem drugą twarz Polski. Gazeta Wyborcza, 115 , S. 10.

Negt, O. (2010). Der politische Mensch. Demokratie als Lebensform. Göttingen: Steidl.

O'Neill, W.F. (1981). Educational ideologies. Contemporary expressions of educational philosophy. Santa Monica CA: Goodyear Publishing Company.

Prawo i Sprawiedliwość (2014). Program PiS 2014. http://pis.org.pl/dokumenty. Zugegriffen: 14. Jan. 2016.

Reporterzy bez Granic piętnuja "dobrą zmianę" w polskich mass mediach. (6. Februar 2017). http:// wiadomosci.onet.pl/swiat/reporterzy-bez-granic-pietnuja-dobra-zmiane-w-polskich-mass-mediach/ zc7g9jh. Zugegriffen: 12.01.2016

Reykowski, J. (2013). Różnice mentalności jako źródła ideologicznych konfliktów. Nauka, 3, 17-43.

Rzepliński, A. (2017). Władza jednostki jest samo dzierżawieniem. http://wiadomosci.onet.pl/tylko-wonecie/andrzej-rzeplinski-wladza-jednostki-jest-samodzierzawiem/khq917v. Zugegriffen: 2. Febr. 2017.

Shell Deutschland (2015). 17. Shell Jugendstudie. Frankfurt a.M.: Fischer.

Śliwerski, B. (2015). Edukacja (w) polityce. Polityka (w) edukacji. Kraków: Oficyna Wydawnicza „Impuls“.

Szafraniec, K. (2011). Młodzi 2011. Warszawa: Kancelaria Prezesa Rady Ministrów.

Sześciło, D., Cześnik, M., \& Markowski, R. (2013). Obywatele $i$ wybory: raport. Warszawa: Fundacja im. Stefana Batorego.

ZNP - Związek Nauczycielstwa Polskiego (2016). Podsumowanie wyników ankiety ZNP ws. Gimnazjów. Warszawa. http://www.znp.edu.pl/element/2572/Podsumowanie_wynikow_ankiety_ZNP_ ws._gimnazjow. Zugegriffen: 12. Jan. 2016. 